\title{
Polymorphism of Human Organic Cationic Transporter1 (C480G) in Egyptian Chronic Myeloid Leukemia Patients on Imatinib
}

\author{
Nahla A. M. Hamed1, Hashim Neanea', Amal M. Ghanem², Maha M. A. Elgammal2, \\ Yasmen Samir ${ }^{2}$ \\ ${ }^{1}$ Hematology Department, Faculty of Medicine, Alexandria University, Alexandria, Egypt \\ ${ }^{2}$ Hematology Department, Medical Research Institute, Faculty of Medicine, Alexandria University, Alexandria, Egypt \\ Email: ys.samir.mri@gmail.com
}

How to cite this paper: Hamed, N.A.M., Neanea, H., Ghanem, A.M., Elgammal, M.M.A. and Samir, Y. (2018) Polymorphism of Human Organic Cationic Transporter1 (C480G) in Egyptian Chronic Myeloid Leukemia Patients on Imatinib. American Journal of Molecular Biology, 8 , 83-91.

https://doi.org/10.4236/ajmb.2018.82007

Received: December 8, 2017

Accepted: April 8, 2018

Published: April 11, 2018

Copyright $\odot 2018$ by authors and Scientific Research Publishing Inc. This work is licensed under the Creative Commons Attribution International License (CC BY 4.0).

http://creativecommons.org/licenses/by/4.0/

\begin{abstract}
Background: Human organic cationic transporter1 (Hoct1) is a plasma membrane transporter responsible for the main influx of Imatinib into chronic myeloid leukemia (CML) cells. Single nucleotide polymorphisms (SNPs) in the gene coding for hOCT1 are important factors causing Imatinib resistance. We investigated the frequency of hOCT1 SNP C480G among Egyptian CML patients and its relation to early molecular response as an indicator of treatment outcome. Materials and Methods: Two groups of CML patients were included in this study. Group I consisted of 25 patients responding to Imatinib treatment (Imatinib responsive) and group II consisted of 25 patients resistant to Imatinib (Imatinib resistant). Response criteria were assessed according to the NCCN (National Comprehensive Cancer Network) guidelines 2017. Twenty healthy controls of matched age and sex were also included (group III). For all patients, we studied hOCT1 C480G at initial presentation using Taqman drug metabolism genotyping as well as BCR-ABL percent at diagnosis and after 3 months interval. Results: hOCT1 C480G was present in $32 \%$ of studied CML patients. CC (wild) was detected in $68 \%$ of group I and $64 \%$ of group II. CG (mutant heterozygous) was present in $28 \%$ of group I and 36\% of group II while GG (mutant homozygous) was detected in only one case in group I. CG was also detected in $15 \%$ of control subjects There was no significant difference between hOCT1 C480G polymorphism and Early Molecular Response $\left(\chi^{2}=0.089, \mathrm{p}=0.765\right)$. Conclusions: hOCT1 C480G polymorphism has no association with Imatinib resistance in Egyptian population. However, further studies on a larger number of patients are still
\end{abstract}


needed to confirm this finding.

\section{Keywords}

Chronic Myeloid Leukemia, Imatinib, Egyptian, Resistance, Human Organic Cationic Transporter1 C480G Polymorphism

\section{Introduction}

CML (Chronic Myeloid Leukemia) is a myeloproliferative neoplasm with an incidence of 1 - 2:100,000 people in developed nations and it is caused by a balanced translocation $\mathrm{t}(9 ; 22)$ ( $\mathrm{q} 34 ; \mathrm{q} 11.2)$ [1]. There is slight male predominance with male to female ratio 1.6. The majority of patients (around 95\%) are diagnosed in CML chronic phase (CML-CP) [2].

Imatinib was the first Tyrosine kinase inhibitor (TKI) licensed and called as "magical bullet", when it revolutionized the treatment of CML [3] [4]. The second generation TKIs Dasatinib and Nilotinib are indicated for treating patients resistant or intolerant to first-line therapy as well as first-line treatment of CML [5]. DASISION and ENESTnd studies have shown that Dasatinib and Nilotinib are associated with higher rates of molecular response and lower risk of disease progression in intermediate and high-risk group [6] [7]. However, Imatinib has clear advantages over the 2nd generation TKIs since its side effect profile is well understood and it is cheaper [8].

The frequency of Imatinib resistance and intolerance is estimated at $30 \%$ $40 \%$ [9]. Resistance to Imatinib may be BCR-ABL1 dependent or independent. Among the mechanisms of BCR-ABL1 independent resistance is the aberrant expressions of drug transporters that may alter intracellular plasma drug concentration [10].

The human Organic Cation Transporter 1 (HOCT1), encoded by the solute carrier 22 family (SLC22A1) gene, is a plasma membrane transporter protein responsible for the translocation of a broad range of drugs [11]. This gene contains 11 exons and 10 introns spanning approximately $37 \mathrm{~kb}$ and localized on chromosome 6q26-7. HOCT1 is primarily found in sinusoidal (basolateral) membranes of hepatocytes and, to a lesser extent, in intestinal cells [12].

Over 1000 SNPs (Single Nucleotide polymorphisms) have been identified, of which 22 have been linked to the treatment outcome of the drugs transported by HOCT1 [13] (Figure 1).

Among the commonest SNPs is hOCT1 C480G (rs683369) in which nucleotide $480 \mathrm{C}$ is changed to $\mathrm{G}$ leading to Leu160Phe replacement [14]. It has a prevalence of about $22 \%$ among Caucasians. This SNP has been found to be correlated with the risk of treatment failure in CML patients [14] [15].

hOCT1 shows genetic heterogeneity in different ethnic groups. Caucasian, African and American (Puerto Ricans, Colombian and Mexican) populations 


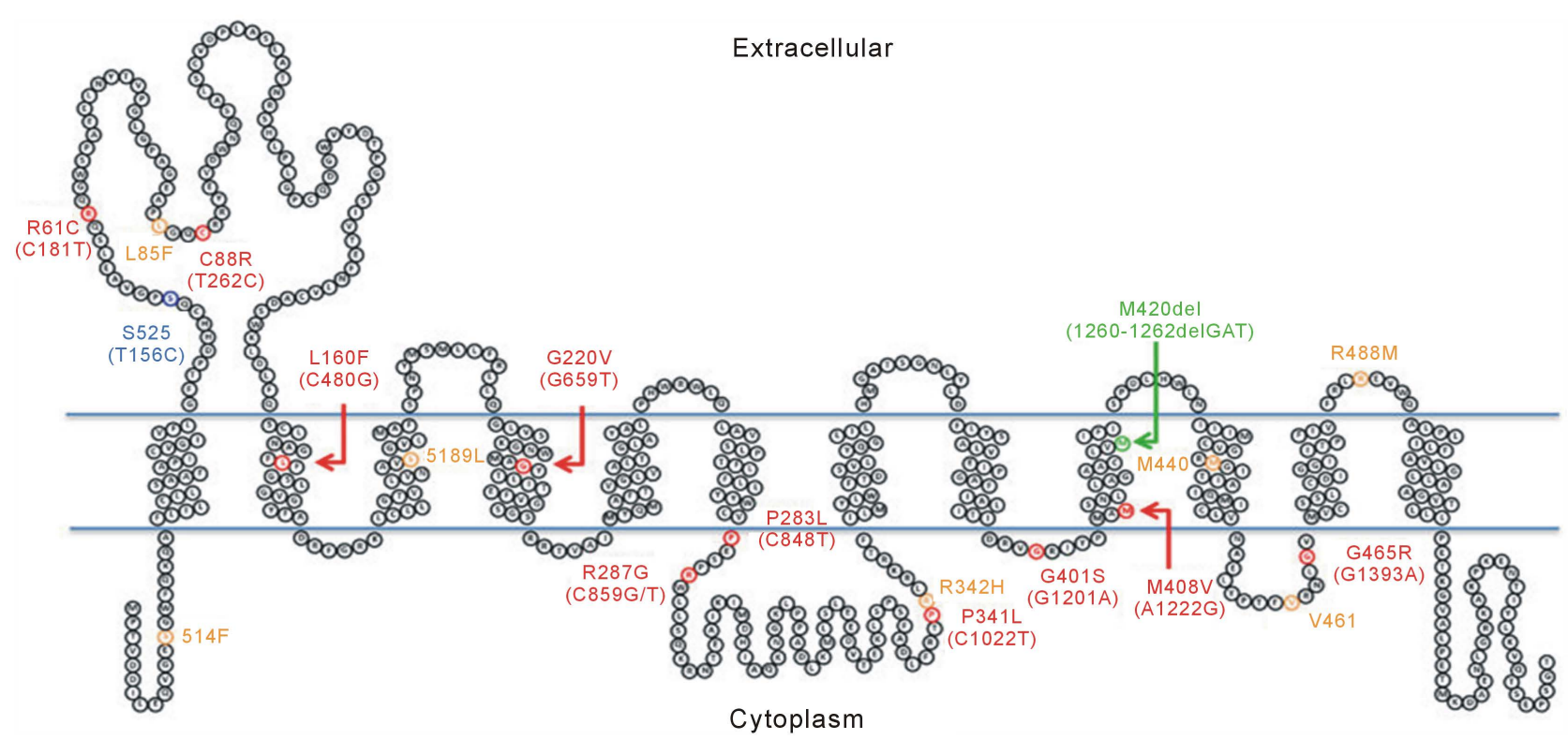

Figure 1. OCT1 and genetic variants with special emphasis on their sites [17].

present higher variability than Asians and Pacific Islanders [13]. The role of hOCT1 polymorphic variants on the pharmacokinetics of Imatinib and its clinical response is controversial.

This study is the first one in Egypt designed to investigate the frequency of hoct1 C480G SNP and its effect on EMR to Imatinib in Egyptian CML patients while there were other studies on hOCT1 were presented by Vine et al. and Paolo et al. [14] [16].

\section{Materials and Methods}

This study included 50 CML patients treated at the Hematology Clinic, Alexandria Main University Hospital and the Medical Research Institute Alexandria University, over 10 months duration between May 2016 and March 2017.The patients were divided according to the early molecular response(EMR) defined as BCR-ABL transcript $\leq 10 \%$ at 3 months of treatment into two groups: group I (Imatinib responsive) consisted of $25 \mathrm{CML}$ patients who achieved EMR and group II (Imatinib resistant) consisted of $25 \mathrm{CML}$ patients who failed to achieve EMR .Response criteria were assessed according to the NCCN guidelines [18].

20 healthy matched subjects were recruited as control group. Risk stratification of CML patients was done using Sokal risk score into low, intermediate and high risk. Patients with hepatic or renal failure, concomitant chronic illness and pregnant females were excluded from the study.

All patients participating in this study were subjected to history taking, clinical examination with special emphasis on spleen size, Complete blood count $(\mathrm{CBC})$, measurement of BCR-ABL1 transcripts at diagnosis and after 3 months of Imatinib treatment by quantitative Reverse transcriptase polymerase chain reaction (RT-PCR) and assessment of the polymorphism of hOCT1 C480G on 
peripheral blood samples by real time PCR using Taqman drug metabolism genotyping assay. Some data were present in the patients' records at the time of the study while other data were conducted at the same time of the study. This study was approved by the local ethics committee at Alexandria University Hospitals, Egypt which goes in accordance with the Helsinki Declaration.

\section{1. hOCT1 C480G SNP Detection}

At first genomic DNA extraction was done using Thermo Scientific Gene JET Genomic DNA Purification Kit. The concentration of DNA was estimated by measuring the absorbance at $260 \mathrm{~nm}$ (A260) using a spectrophotometer (Nanodrop $^{\circledR}$ ND-1000 spectrophotometer). DNA purity was estimated from the A260/A280 ratio. An A260/A280 ratio between 1.7 and 2.0 generally represents a high-quality DNA sample.

Samples were stored at $-20^{\circ} \mathrm{C}$. The following primer was used AGTCCTGTT TGAATGCGGGCTTCTT[C/G]TTTGGCTCTCTCGGTGTTGGCTACT. Primers were validated through NCBI blast. PCR reaction mix was performed using Taqman drug metabolism genotyping assay. Analysis of the SNP for hOCT1 C480G (rs683369) was done by Real time PCR. Thermocycling conditions were $10 \mathrm{~min}$ at $95^{\circ} \mathrm{C}$, followed by 40 cycles of denaturation $\left(95^{\circ} \mathrm{C}, 15 \mathrm{sec}\right)$. Results were divided into CC (wild type) CG (mutant heterozygous) and GG (mutant homozygous).

\subsection{Measurement of BCR-ABL1 Transcripts}

Total RNA was extracted from peripheral blood mononuclear cells using RNeasy Midi Kit (Qiagen) and was converted into cDNA using ipsogen RT kit (Qiagen). RQ-PCR was analysed through Rotor-gene $Q$ instrument using ipsogen BCR-ABL1 kit (Qiagen). The absolute quantities of BCR-ABL and ABL transcripts in patient specimens were determined by reference to standard curves [19].

\subsection{Statistical Analysis}

Data were analyzed using IBM Statistical Package for the Social Sciences (SPSS) (version 24.0; SPSS Inc., Chicago, IL, USA). Data were tested for normality using Kolmogorov-Smirnov test, Shapiro-Wilk test. Mean and standard deviation were used to describe the scale data while frequency was used to describe the categorical data. Parametric data were analyzed using t test to compare 2 means and ANOVA(f) test to compare more than 2 means. Non-parametric data were analyzed using Mann Whitney and Kruskal Wallis tests. Qualitative data such as sex, Sokal score, genotype distribution and frequencies were presented by percentages and tested by Chi Square $\chi^{2}$ and Fisher Exact Test according to the categories and cells' estimation percent when more than $20 \%$ of the cells have expected count $<5$. A difference was considered significant if $p$ value was less than 0.05 in all analyses. 


\section{Results}

Baseline characteristics including age, sex, spleen size, Sokal score and Bcr-ABL1 transcripts at 3 months are presented in Table 1 and Table 2.

The distribution of hOCT1 C480G genotypes in both CML and control groups is shown in Table 1. In group I, 68\% (17 patients) were wild homozygous CC, $28 \%$ (seven patients) were heterozygous CG and $4 \%$ (one patient) was homozygous (mutant) CG. However, in group II, 64\% (16 patients) were CC and $36 \%$ (nine patients) were CG. In controls, $85 \%$ (17 patients) were CC and 15\% (3 patients) were CG. There was no statistically significant difference among the three studied groups $\left(\chi^{2}=4.215, \mathrm{p}=0.312\right)$.

Table 1. Comparison of CML patients and control cases regarding age, sex and C480G genotypes.

\begin{tabular}{|c|c|c|c|c|c|c|c|c|}
\hline \multirow{3}{*}{ Parameter } & \multicolumn{6}{|c|}{ Groups $(n=70)$} & \multirow{3}{*}{$\begin{array}{l}\text { Test of } \\
\text { sig. }\end{array}$} & \multirow{3}{*}{$\mathbf{P}$} \\
\hline & \multicolumn{2}{|c|}{$\begin{array}{l}\text { Group I } \\
(\mathrm{n}=25)\end{array}$} & \multicolumn{2}{|c|}{$\begin{array}{l}\text { Group II } \\
(\mathrm{n}=25)\end{array}$} & \multicolumn{2}{|c|}{$\begin{array}{l}\text { Control } \\
(\mathrm{n}=20)\end{array}$} & & \\
\hline & No. & $\%$ & No. & $\%$ & No. & $\%$ & & \\
\hline \multicolumn{9}{|l|}{ Sex } \\
\hline Male & 12 & 48 & 14 & 56 & 11 & 55 & $\chi^{2}=0.373$ & 0.830 \\
\hline Female & 13 & 52 & 11 & 44 & 9 & 45 & & \\
\hline Age (years) & \multicolumn{2}{|c|}{$43.7 \pm 13.4$} & \multicolumn{2}{|c|}{$48.64 \pm 16.77$} & \multicolumn{2}{|c|}{$39.1 \pm 12.03$} & $F=2.463$ & 0.093 \\
\hline \multicolumn{9}{|l|}{ Genotype } \\
\hline $\mathrm{CC}$ & \multicolumn{2}{|c|}{$17(68 \%)$} & \multicolumn{2}{|c|}{$16(64 \%))$} & \multicolumn{2}{|c|}{$17(85 \%)$} & \multirow{3}{*}{$\chi^{2}=4.21$} & \multirow{3}{*}{0.312} \\
\hline CG & \multicolumn{2}{|c|}{$7(28 \%)$} & \multicolumn{2}{|c|}{$9(36 \%)$} & \multicolumn{2}{|c|}{$3(5 \%)$} & & \\
\hline GG & \multicolumn{2}{|c|}{$1(4 \%)$} & \multicolumn{2}{|c|}{$0(0 \%)$} & \multicolumn{2}{|c|}{$0(0 \%)$} & & \\
\hline
\end{tabular}

Group I: Imatinib responsive, Group II: Imatinib resistant; CC (wild), CG (mutant heterozygous), GG (mutant homozygous).

Table 2. Clinical and laboratory parameters among CML patients.

\begin{tabular}{ccccc}
\hline Parameter & $\begin{array}{c}\text { Group I } \\
(\mathrm{n}=25)\end{array}$ & $\begin{array}{c}\text { Group II } \\
(\mathrm{n}=25)\end{array}$ & Test of sig & $\mathrm{P}$ \\
\hline Spleen size $(\mathrm{cm})$ & $20.78 \pm 4.2$ & $25.10 \pm 3.6$ & $\mathrm{t}=3.770$ & $0.001^{*}$ \\
Sokal score & $1.21 \pm 0.342$ & $1.5 \pm 0.36$ & $\mathrm{t}=2.819$ & $0.002^{*}$ \\
$\begin{array}{c}\text { Sokal risk category } \\
\text { Low risk }\end{array}$ & $4(16 \%)$ & - & & \\
Intermediate & $10(40 \%)$ & $5(20.8 \%)$ & $\chi^{2}=7.402$ & $0.023^{*}$ \\
High & $11(44 \%)$ & $19(79.2 \%)$ & & \\
BCR-ABL1 at & $70.37 \pm 26.25$ & $73.88 \pm 22.85$ & $\mathrm{u}=295.000$ & 0.731 \\
diagnosis $(\%)$ & & & & \\
BCR-ABL1 transcript & $4.53 \pm 5.23$ & $35.54 \pm 8.13$ & $\mathrm{u}=14.500$ & $0.000^{*}$ \\
at 3 months $(\%)$ & & & & \\
C480G genotype & & & & \\
CC & $17(51.5 \%)$ & $9(53.0 \%)$ & & \\
CG+GG & $8(47 \%)$ & & & \\
\hline
\end{tabular}

Group I (Imatinib responsive), group II (Imatinib resistant); CC wild, CG mutant heterozygous, GG mutant homozygous; ${ }^{\star} \mathrm{P}$ is significant when $\leq 0.05$. 
When categorizing CML patients according to the Sokal risk score and C480G polymorphism, it was found that in group I, patients having the CC genotype were $23.5 \%$ (four patients) low risk Sokal, $47.1 \%$ (8 patients) were intermediate risk and $29.5 \%$ (five patients) were high risk whereas patients with the CG or GG genotype were 25\% (two patients) intermediate risk and 75\% (six patients) were high risk. In group II, patients carrying the CC genotype were $26.7 \%$ (four patients) intermediate risk and $73.3 \%$ (11 patients) were high risk whereas patients who had the CG genotype, $11.1 \%$ (one patient) was intermediate risk and $88.9 \%$ (eight patients) were high risk. Sokal score was significantly higher among group II especially patients carrying the CG genotype $\left(\chi^{2}=11.954, \mathrm{p}=0.026\right)$.

There is no significant difference in age $(\mathrm{p}=0.463)$, sex $(\mathrm{p}=0.709)$, BCR-ABL1 at diagnosis $(p=0.902), \operatorname{EMR}(p=0.765)$, Hb level $(p=0.726)$, WBCs $(\mathrm{p}=0.197)$ and platelets count $(\mathrm{p}=0.990)$ among the CC, CG and GG. There was significant difference among hOCT1 C480G genotypes and spleen size $(\mathrm{p}=0.001)$ in the CML studied groups (Table 3$)$.

\section{Discussion}

This study aimed at investigating the frequency of the polymorphism of Hoct1 transporter C480G and EMR. The present study couldn't find a statistically significant effect of hOCT1 polymorphism on the EMR. In accordance with our results, Takahashi et al. studied hOCT1 C480G polymorphism among other polymorphisms and their effects on Imatinib drug concentration and clinical response. They found no association between hOCT1 C480G polymorphism and molecular response [20].

Table 3. Comparison of hOCT1 C480G and different clinical and lab parameters.

\begin{tabular}{|c|c|c|c|c|c|c|}
\hline \multirow[b]{2}{*}{ Parameters } & \multicolumn{2}{|c|}{ Group I } & \multicolumn{2}{|c|}{ Group II } & \multirow[b]{2}{*}{ Test of Sig. } & \multirow[b]{2}{*}{$\mathrm{P}$} \\
\hline & $\begin{array}{c}\text { CC } \\
(n=17)\end{array}$ & $\begin{array}{c}C G+G G \\
(n=8)\end{array}$ & $\begin{array}{c}\text { CC } \\
(n=16)\end{array}$ & $\begin{array}{c}\text { CG } \\
(\mathrm{n}=9)\end{array}$ & & \\
\hline Age (years) & $41.82 \pm 12.17$ & $47.88 \pm 15.90$ & $50.25 \pm 17.92$ & $45.78 \pm 15.1$ & $\mathrm{~F}=0.871$ & 0.463 \\
\hline \multicolumn{7}{|l|}{ Sex } \\
\hline Male & $8(47.1 \%)$ & $5(62.5 \%)$ & $6(37.5 \%)$ & $5(55.6 \%)$ & \multirow{2}{*}{$\chi^{2}=1.645$} & \multirow[t]{2}{*}{0.709} \\
\hline Female & $9(52.9 \%)$ & $3(37.5 \%)$ & $10(32.5 \%)$ & $4(44.4 \%)$ & & \\
\hline Spleen size $(\mathrm{cm})$ & $20.28 \pm 4.08$ & $22.13 \pm 4.49$ & $24.03 \pm 3.20$ & $26.89 \pm 3.72$ & $\mathrm{~F}=6.437$ & $0.001^{\star}$ \\
\hline \multicolumn{7}{|l|}{ Sokal score } \\
\hline Low & $4(23.5 \%)$ & 0 & 0 & 0 & \multirow{3}{*}{$\chi^{2}=11.954$} & \multirow{3}{*}{$0.026^{*}$} \\
\hline Intermediate & $8(47.1 \%)$ & $2(25 \%)$ & $4(26.7 \%)$ & $1(11.1 \%)$ & & \\
\hline High & $5(29.4)$ & $6(75 \%)$ & $11(73.3 \%)$ & $8(88.9 \%)$ & & \\
\hline Hemoglobin (g/dl) & $10.61 \pm 1.46$ & $9.74 \pm 2.56$ & $10.28 \pm 2.04$ & $9.93 \pm 2.18$ & $\mathrm{~F}=0.440$ & 0.726 \\
\hline Platelets $\left(\times 10^{9} / \mathrm{L}\right)$ & $351.8 \pm 177.2$ & $384.3 \pm 226.5$ & $372.3 \pm 264.5$ & $373.6 \pm 233.4$ & $\mathrm{H}=0.116$ & 0.990 \\
\hline WBCs $\left(\times 10^{9} / \mathrm{L}\right)$ & $187.6 \pm 163.9$ & $249.6 \pm 178.1$ & $114.1 \pm 109.4$ & $155.2 \pm 125.6$ & $\mathrm{H}=4.676$ & 0.197 \\
\hline BCR-ABL1 at diagnosis (\%) & $69.96 \pm 25.14$ & $73.29 \pm 32.12$ & $72.12 \pm 21.16$ & $77.0 \pm 26.63$ & $\mathrm{H}=0.484$ & 0.902 \\
\hline $\mathrm{EMR}^{* *}$ & \multicolumn{2}{|c|}{$\leq 10 \%$} & \multicolumn{2}{|c|}{$>10 \%$} & $\chi^{2}=0.089$ & 0.765 \\
\hline
\end{tabular}

Group I (Imatinib responsive), group II (Imatinib resistant); CC (wild), CG (mutant heterozygous), GG (mutant homozygous); ${ }^{*} \mathrm{p}$ significant if $\leq 0.05$; ${ }_{* *}^{*}$ EMR: Early molecular response. 
Similarly, Vine et al. investigated the relation between hOCT1 SNPs (C480G, Met408Val) and multidrug resistance gene (MDR1) SNP and Imatinib response using restriction enzyme analysis and correlated them with Imatinib response and Imatinib drug level. hOCT1 polymorphisms did not predict treatment failure when correlated with Imatinib levels. Patients with GG C480G genotype needed a slightly higher Imatinib level to achieve molecular response but this wasn't statistically significant [16]. Similarly, De Lima et al. found that hOCT1 mRNA expression rather than hOCT1 SNPs may be associated with Imatinib response. They found no correlation between C480G and hematological, cytogenetic and molecular response [21]. Koren-Michowitz $\mathrm{M}$ studied the effect of hOCT1 genetic variants on long-term outcomes of Imatinib treated patients and analyzed many SNPs in hOCT1 using the Sequenom Mass ARRAY platform and C480G was one of these variants. Rates of molecular responses were not significantly different according to allelic variant [22].

Contradictory results were reported by Paolo et al. who found that hOCT1 C480G may be associated with a reduced intracellular uptake of Imatinib with the following increased risk of treatment failure. Paolo et al. correlated Imatinib drug concentration with the presence of the hOCT1 C480G and Imatinib response [15]. Ankathil et al. found that genetic variation $C \rightarrow G$ might lead to a decrease in enzymatic activity that results in an inadequate response to treatment. Carriers of the G allele of hOCT1 C480G, might be at increased risk of acquiring resistance [23] Kim et al. found that the hOCT1 GG genotype is correlated with high rate of loss of response or treatment failure to Imatinib therapy whereas the CC and CG genotypes are associated with treatment response [24]. Cao et al. assessed HOCT1 expression level in granulocytes using indirect immunofluorescence and flow cytometry. This study revealed that hOCT1 expression was significantly associated with Imatinib efficacy and achievement of MMR (Major molecular response) [25]. The difference and contradiction in the results may be related to the different sample sizes, population under study, different methodology used and other parameters that could affect Imatinib response (namely Imatinib drug level, Hoct1 activity and gene expression).

In the present study, Sokal score was significantly advanced in group II patients especially those with the CG genotype $(\mathrm{p}=0.026)$. This results are more or less similar to Chhikara et al. who reported that patients with high OCT1 expression had significant higher Sokal scores than those with low OCT1 expression [25].

Although the present work couldn't find a significance impact of C490G polymorphism on EMR, BCR-ABL1 transcript level at 3 months were significantly higher in group II especially those having the heterozygous form (CG). CG genotype may be associated with a higher risk evidenced in this study by the statistically more advanced Sokal score and higher BCR-ABL transcripts at 3 months.

\section{Conclusion}

From this study, it was concluded that C480G polymorphism is present in Egyp- 
tian CML patients. EMR was not affected by the presence of C480G polymorphism in CML patients. Spleen size and Sokal score were significantly higher in patients carrying the mutant (CG) than those with normal wild (CC). So, C480G polymorphism may be associated with higher risk. Testing these findings in a larger patient population with newly diagnosed CML is recommended.

\section{References}

[1] Jabbour, E. and Kantarjian, H. (2016) Chronic Myeloid Leukemia: 2016 Update on Diagnosis, Therapy, and Monitoring. American Journal of Hematology, 91, 252-265. https://doi.org/10.1002/ajh.24275

[2] Siegel, R.L., Miller, K.D. and Jemal, A. (2017) Cancer Statistics, 2017. CA: A Cancer Journal for Clinicians, 67, 7-30. https://doi.org/10.3322/caac.21387

[3] Yeung, D.T. and Hughes, T.P. (2016) Chronic Myeloid Leukemia. In: Hoffbrand, A.V., Higgs, D.R., Keeleg, M.M. and Mehta, A.B., Eds., Postgraduate Hematology, 7th Edition, John Wiley \& Sons Ltd., Wiley Blackwell, 419-437.

[4] Iqbal, N. and Iqbal, N. (2014) Imatinib: A Breakthrough of Targeted Therapy in Cancer. Chemotherapy Research and Practice, 2014, Article ID: 357027. https://doi.org/10.1155/2014/357027

[5] Jabbour, E., Kantarjian, H. and Cortes, J. (2015) Use of Second- and Third-Generation Tyrosine Kinase Inhibitors in the Treatment of Chronic Myeloid Leukemia: An Evolving Treatment Paradigm. Clinical Lymphoma, Myeloma and Leukemia, 15, 323-334. https://doi.org/10.1016/j.clml.2015.03.006

[6] Cortes, J.E., Saglio, G., Kantarjian, H.M., Baccarani, M., Mayer, J., Boqué, C., et al. (2016) Final 5-Year Study Results of DASISION: The Dasatinib versus Imatinib Study in Treatment-Naïve Chronic Myeloid Leukemia Patients Trial. Journal of Clinical Oncology, 34, 2333-2340. https://doi.org/10.1200/JCO.2015.64.8899

[7] Hochhaus, A., Saglio, G., Hughes, T.P., Larson, R.A., Kim, D.W., Issaragrisil, S., et al. (2016) Long-Term Benefits and Risks of Frontline Nilotinib vs Imatinib for Chronic Myeloid Leukemia in Chronic Phase: 5-Year Update of the Randomized ENESTnd Trial. Leukemia, 30, 1044-1054. https://doi.org/10.1038/leu.2016.5

[8] Neelakantan, P., Gerrard, G., Lucas, C., Milojkovic, D., May, P., Wang, L., et al. (2013) Combining BCR-ABL1 Transcript Levels at 3 and 6 Months in Chronic Myeloid Leukemia: Implications for Early Intervention Strategies. Blood, 121, 2739. https://doi.org/10.1182/blood-2012-11-466037

[9] Khorashad, J.S., Kelley, T.W., Szankasi, P., Mason, C.C., Soverini, S., Adrian, L.T., et al. (2012) BCR-ABL1 Compound Mutations in Tyrosine Kinase Inhibitor-Resistant CML: Frequency and Clonal Relationships. Blood, 121, 489-498. https://doi.org/10.1182/blood-2012-05-431379

[10] Thomas, J. (2004) Active Transport of Imatinib into and Out of Cells: Implications for Drug Resistance. Blood, 104, 3739-3745. https://doi.org/10.1182/blood-2003-12-4276

[11] Arimany-Nardi, C., Minuesa, G., Keller, T., Erkizia, I., Koepsell, H., Martinez-Picado, J., et al. (2016) Role of Human Organic Cation Transporter 1 (hOCT1) Polymorphisms in Lamivudine (3TC) Uptake and Drug-Drug Interactions. Frontiers in Pharmacology, 7, 175. https://doi.org/10.3389/fphar.2016.00175

[12] Marin, J.J.G. (2012) Plasma Membrane Transporters in Modern Liver Pharmacology. Scientifica, 2012, Article ID: 428139. https://doi.org/10.6064/2012/428139

[13] Arimany-Nardi, C., Koepsell, H. and Pastor-Anglada, M. (2015) Role of SLC22A1 Polymorphic Variants in Drug Disposition, Therapeutic Responses, and Drug-Drug 
Interactions. The Pharmacogenomics Journal, 15, 473-487. https://doi.org/10.1038/tpj.2015.78

[14] Di Paolo, A., Polillo, M., Capecchi, M., Cervetti, G., Baratè, C., Angelini, S., et al. (2014) The c.480C > G Polymorphism of hOCT1 Influences Imatinib Clearance in Patients Affected by Chronic Myeloid Leukemia. The Pharmacogenomics Journal, 14, 328-335. https://doi.org/10.1038/tpj.2014.7

[15] Maffioli, M., Camós, M., Gaya, A., Hernández-Boluda, J.-C., Álvarez-Larrán, A., Domingo, A., et al. (2011) Correlation between Genetic Polymorphisms of the hOCT1 and MDR1 Genes and the Response to Imatinib in Patients Newly Diagnosed with Chronic-Phase Chronic Myeloid Leukemia. Leukemia Research, 35, 1014-1019. https://doi.org/10.1016/j.leukres.2010.12.004

[16] Vine, J., Cohen, S.B., Ruchlemer, R., Goldschmidt, N., Levin, M., Libster, D., et al. (2014) Polymorphisms in the Human Organic Cation Transporter and the Multidrug Resistance Gene: Correlation with Imatinib Levels and Clinical Course in Patients with Chronic Myeloid Leukemia. Leukemia \& Lymphoma, 55, 2525-2531. https://doi.org/10.3109/10428194.2014.893307

[17] Watkins, D.B., Hughes, T.P. and White, D.L. (2015) OCT1 and Imatinib Transport in CML: Is It Clinically Relevant? Leukemia, 29, 1960-1969.

[18] National Comprehensive Cancer Network (2018) Chronic Myeloid Leukemia. https://www.nccn.org/professionals/physician_gls/PDF/cml.pdf

[19] Foroni, L., Wilson, G., Gerrard, G., Mason, J., Grimwade, D., White, H.E., et al. (2011) Guidelines for the Measurement of BCR-ABL1 Transcripts in Chronic Myeloid Leukaemia. British Journal of Haematology, 153, 179-190. https://doi.org/10.1111/j.1365-2141.2011.08603.x

[20] Takahashi, N., Miura, M., Scott, S.A., Kagaya, H., Kameoka, Y., Tagawa, H., et al. (2010) Influence of CYP3A5 and Drug Transporter Polymorphisms on Imatinib Trough Concentration and Clinical Response among Patients with Chronic Phase Chronic Myeloid Leukemia. Journal of Human Genetics, 55, 731-737. https://doi.org/10.1038/jhg.2010.98

[21] de Lima, L.T., Vivona, D., Bueno, C.T., Hirata, R.D.C., Hirata, M.H., Luchessi, A.D., et al. (2014) Reduced ABCG2 and Increased SLC22A1 mRNA Expression Are Associated with Imatinib Response in Chronic Myeloid Leukemia. Medical Oncology, 31, 851. https://doi.org/10.1007/s12032-014-0851-5

[22] Koren-Michowitz, M., Buzaglo, Z., Ribakovsky, E., Schwarz, M., Pessach, I., Shimoni, A., et al. (2013) OCT1 Genetic Variants Are Associated with Long Term Outcomes in Imatinib Treated Chronic Myeloid Leukemia Patients. European Journal of Hematology, 92, 283-288. https://doi.org/10.1111/ejh.12235

[23] Ankathil, R., Siti Maziras, M., Ahmad Aizat, A.A., Au Zian, A. and Azlan, H. (2014) OP0025 Genetic Association of C480G Polymorphism of SLC22A1 with Clinical Resistance to Imatinib Mesylate in Malaysian Patients with Chronic Myeloid Leukaemia. European Journal of Cancer, 50, e9.

[24] Kim, D.H., Sriharsha, L., Xu, W., Kamel-Reid, S., Liu, X., Siminovitch, K., et al. (2009) Clinical Relevance of a Pharmacogenetic Approach Using Multiple Candidate Genes to Predict Response and Resistance to Imatinib Therapy in Chronic Myeloid Leukemia. Clinical Cancer Research, 15, 4750. https://doi.org/10.1158/1078-0432.CCR-09-0145

[25] Chhikara, S., Sazawal, S., Seth, T., Chaubey, R., Singh, K., Sharma, R., et al. (2017) Molecular Response to Imatinib and Its Correlation with mRNA Expression Levels of Imatinib Influx Transporter (OCT1) in Indian Chronic Myeloid Leukemia Patients. Asian Pacific Journal of Cancer Prevention, 18, 2043-2048. 